\title{
Impacto psicológico estado de ansiedad y depresión en trabajadores de salud frente al COVID-19
}

Psychological impact of anxiety and depression in health care workers in the face of COVID-19

Estado de impacto psicológico de ansiedade e depressão em trabalhadores do setor de saúde diante da COVID-19

Rosa María Zambrano Garcés

rmzambranog@ucacue.edu.ec https://orcid.org/0000-0002-8846-148X

\section{Luis Cornelio Romero Mogrovejo}

tromerom@ucacue.edu.ec

https://orcid.org/0000-0002-0475-5174

\author{
enriqueta.martinez@ucacue.edu.ec \\ https://orcid.org/0000-0003-3733-2458 \\ Andrea Vicuña Palacios \\ andreavicu29@gmail.com \\ https://orcid.org/0000-0002-5603-1872
}

Enriqueta de los Ángeles Martínez Vicuña

\author{
Universidad Católica de Cuenca, Cuenca-Ecuador \\ Recibido 13 de octubre 2021 / Arbitrado y aceptado 16 de noviembre 2021 / Publicado 30 de diciembre 2021
}

\begin{abstract}
RESUMEN
La pandemia por COVID-19 generó un gran impacto en la salud mental en los profesionales de salud y más aún por los que trabajan en primera línea. El objetivo principal fue conocer el impacto psicológico estado ansiedad y depresión en trabajadores de salud frente al covid-19 en el cantón La Troncal. Se realizó un estudio descriptivo, corte transversal, correlativo, comparativo, con enfoque cuantitativo, los datos fueron secundarios $(n=100)$, se evaluó la Escala de Ansiedad y Depresión de Goldberg (GADS). Se realizó un análisis descriptivo mediante porcentajes, frecuencias, medidas de tendencia central y percentiles, también se hizo un análisis de diferencia de medias de dos grupos mediante la prueba t de Student para muestras independientes y el coeficiente de correlación de Pearson (r), encontrando edad media de la población es 34.57 años, tiempo medio que labora dentro de la institución 4.63 años, participó mayoritariamente el género femenino $(n=70)$, Enfermeras $(n=37)$, lugar de trabajo en iguales condiciones. En cuanto a la ansiedad está presente $(n=81)$, pero la depresión está ausente $(n=89)$. En género tienen igual ansiedad y depresión, el centro de salud Tipo $C$ tiene mayor nivel de ansiedad. Y entre la ansiedad y el tiempo que labora una relación negativa $(r=-0.2)$, es decir a mayor tiempo que labora dentro de la institución menor es el nivel de ansiedad. En conclusión, se debe realizar programas de intervención para disminuir los problemas de salud mental en los profesionales del cantón la Troncal.
\end{abstract}

Palabras clave: Ansiedad; Depresión; Trabajadores de la salud; Covid-19

\begin{abstract}
The COVID-19 pandemic generated a great impact on the mental health of health professionals and even more so for those working on the front line. The main objective was to know the psychological impact of the state of anxiety and depression in health workers in the canton of La Troncal. A descriptive, cross-sectional, correlative, comparative, quantitative, crosssectional study was carried out with a quantitative approach, the data were secondary $(n=100)$, and the Goldberg Anxiety and Depression Scale (GADS) was evaluated. A descriptive analysis was carried out using percentages, frequencies, measures of central tendency and percentiles, an analysis of the difference of means of two groups was also made using Student's t-test for independent samples and Pearson's correlation coefficient ( $r$ ), finding that the mean age of the population was 34.57 years, mean time working in the institution was 4.63 years, the majority of participants were women $(n=70)$, nurses $(n=37)$, workplace in the same conditions. Anxiety is present $(n=81)$, but depression is absent $(n=89)$. In gender have equal anxiety and depression, Type $C$ health center has higher level of anxiety. There is a negative relationship $(r=-0.2)$ between anxiety and working time, i.e. the longer the time spent working in the institution, the lower the level of anxiety. In conclusion, intervention programs should be carried out to reduce mental health problems among professionals in the canton of La Troncal.
\end{abstract}

Key words: Anxiety; Depression; Health care workers; Covid-19 


\section{RESUMO}

A pandemia COVID-19 teve um grande impacto sobre a saúde mental dos profissionais de saúde, especialmente aqueles que trabalham na linha de frente. O principal objetivo era determinar o impacto psicológico da covid-19 nos trabalhadores da saúde no cantão de La Troncal em termos de ansiedade e depressão. Um estudo descritivo, transversal, correlacional, comparativo, quantitativo, transversal foi realizado com uma abordagem quantitativa, os dados eram secundários $(n=100)$, e a Escala de Ansiedade e Depressão Goldberg (GADS) foi avaliada. Uma análise descritiva foi realizada utilizando porcentagens, freqüências, medidas de tendência central e percentis, uma análise da diferença entre as médias de dois grupos também foi realizada utilizando o teste t de Student para amostras independentes e o coeficiente de correlação de Pearson ( $r$ ), encontrando a idade média da população em 34,57 anos, o tempo médio de trabalho na instituição em 4,63 anos, a maioria dos participantes eram mulheres $(n=70)$, enfermeiras $(n=37)$, e o local de trabalho estava nas mesmas condições. A ansiedade está presente $(n=81)$, mas a depressão está ausente $(n=89)$. No gênero, eles têm ansiedade e depressão iguais, o centro de saúde tipo $C$ tem um nível mais alto de ansiedade. Existe uma relação negativa entre ansiedade e tempo de trabalho $(r=-0,2)$, ou seja, quanto maior o tempo de trabalho na instituição, menor é o nível de ansiedade. Em conclusão, devem ser implementados programas de intervenção para reduzir os problemas de saúde mental entre os profissionais do cantão de La Troncal.

Palavras-Chave: Ansiedade; Depressão; Trabalhadores da Saúde; Covid-19

\section{INTRODUCCIÓN}

Una nueva enfermedad de rápido contagio causada por la cepa viral SARS-CoV-2 que apareció en diciembre del 2019 en Wuhan, China; causando alarma y estrés en la población, de manera especial en el personal de salud. El miedo hacia lo desconocido, noticias erróneas, desinformación por medios de comunicación y redes sociales, el incremento de casos confirmados y fallecimientos por covid-19 provocaron emociones negativas como: ansiedad, depresión, miedo, intentos auto líticos, entre otros (1).
A nivel mundial según la OMS hasta abril del 2021 existen 56.759.442 casos confirmados, fallecimientos 1.372.642, en Ecuador 335.681 casos confirmados y 16.956 fallecimientos por Covid-19 (2). El covid-19 produce síntomas que van desde un cuadro febril hasta un síndrome respiratorio agudo, llegando a desenlaces fatales. Desde el 29 febrero del 2020 que el Ministerio de Salud Pública del Ecuador confirma el primer caso por covid-19, se inició la emergencia sanitaria dando consecuencias negativas en la salud mental (3). El personal de salud al estar en primera línea fue quizás el grupo más afectado por la falta de conocimiento, de prendas de protección, horarios laborales extensos, el aislamiento de sus familiares, el miedo al incremento continuo de la curva de contagio y muertes, volviéndolos más vulnerables desde la perspectiva psicológica $(4,5)$.

El sector económico y social de los países se han visto afectados frente a la pandemia por covid-19 y los sistemas de salud son los más afectados pues a pesar de las múltiples estrategias y recursos empleados para controlar la propagación de este virus y disminuir su impacto en la salud mental, se ha presentado en todos los países. Las medidas tomadas como el aislamiento y distanciamiento social han aumentado el nivel de estrés, ansiedad y miedo; además ha afectado la economía de las empresas colocándolas en situaciones críticas generando desempleo y por ende dificultades económicas en las familias y personas, aumentando aún más el riesgo de alteraciones psicológicas (6). 
Un estudio realizado al personal de salud en China con 246 participantes (médicos 34.4\% y enfermeras 69.6\%) donde se aplicó la escala de autoevaluación para la ansiedad (SAS) y la escala de autoevaluación para el trastorno de estrés postraumático (PTSD-SS), mostrando una tasa de ansiedad del $23.04 \%$, las enfermeras presentaron mayor nivel de ansiedad en comparación de los médicos $26.88 \%$ frente a $14.29 \%$. En cuanto al trastorno de estrés el personal de salud presentó una tasa de $27.39 \%$, siendo más evidente en el grupo femenino con $44.33 \%$, mientras que el gripo masculino presentó un $36.91 \%$. Llegando a la conclusión que las casas de salud deben implementar acciones de intervención psicológica para controlar y mejorar el impacto psicológico de la pandemia en el personal de salud prestando mayor atención en el personal de enfermería (7).

Luego de la nueva realidad en la que vivimos por covid-19, donde tendremos que mantener el distanciamiento social, lavado de manos frecuente, evitar la aglomeración social, entre otras medidas, donde el mundo no volverá a ser el mismo según estudios (8), surge la pregunta ¿Existe impacto psicológico estado de ansiedad y depresión en los trabajadores de salud frente al covid-19 en el cantón la Troncal? El objetivo general es conocer el impacto psicológico estado ansiedad y depresión en los trabajadores de salud frente al covid-19 en el cantón La Troncal.

Se plantearon los siguientes objetivos específicos: 1) Describir las características sociodemográficas de la población estudio,
2 Comparar el estado ansiedad y depresión con el género y lugar de trabajo y 3) Analizar el tiempo que labora en la institución con la ansiedad y depresión.

\section{MATERIALES Y MÉTODOS}

Se realizó un estudio descriptivo, de corte transversal, correlativo, comparativo, con enfoque cuantitativo, los datos trabajados fueron secundarios tomados del departamento de psicología del Distrito de Salud 03D03 La Troncal, quienes aplicaron al personal de salud una encuesta validada Escala de Ansiedad y Depresión de Goldberg (GADS). La población fue finita de 100 historias clínicas del departamento de psicología del Distrito de Salud 03D03 La Troncal. Se incluyó al personal de salud operativo del Hospital Darío Machuca Palacios o Centro de Salud Tipo $C$ que el psicólogo del Distrito de Salud 03D03 la Troncal haya aplicado la escala GADS. Y se excluyó a personal de salud en funciones administrativas, o que no pertenezcan a las instituciones mencionadas-, demográficamente fue evaluado las variables edad, género, profesión y lugar de trabajo.

El instrumento que se usó sobre Escala de Ansiedad y Depresión de Goldberg (GADS), es una escala que no solo orienta al diagnóstico de ansiedad o depresión o de ambos sino también discrimina entre ellos y dimensiona sus intensidades. Consta de 2 subescalas ansiedad (preguntas 1 a la 9) y depresión (preguntas de 10 a la 18), consta de escala dicotómica y se califica para la subescala ansiedad 4 o más 
puntos y en depresión 2 puntos o más, a mayor puntuación más severo es el problema (9).

\section{Procedimiento}

Los datos se tomaron de las historias clínicas de psicología del Distrito de Salud 030D03 la Troncal de los trabajadores de salud del Hospital Darío Machuca Palacios como del Centro de Salud Tipo C. Los datos tanto de las variables sociodemográficas como de la escala GADS fueron tabuladas en una página Excel, el formato compatible con ASCII. Además, se elaboró un libro de códigos para mejorar la operacionalización y posterior análisis. Los datos de los participantes son confidenciales no se tomó de las historias clínicas nombre ni números de cédula. Los procedimientos estadísticos fueron programados en $R$ (se facilitarán los scripts). Con el mismo programa (software libre) se realizaron lo correspondientes análisis estadísticos.

\section{Consideraciones éticas}

Se tuvo el consentimiento informado de parte de las autoridades del Centro los trabajadores de salud del Hospital Darío Machuca Palacios como del Centro de Salud Tipo C; así como de la población estudiada.

\section{Análisis estadístico}

Se realizó un análisis descriptivo mediante porcentajes, frecuencias(variablescualitativas), medidas de tendencia central y percentiles (variables cuantitativas), posteriormente se realizará una prueba de normalidad mediante Shapiro Wilk (W) y Homocedasticidad (Prueba de Levene). Se efectuó un análisis de diferencia de medias de dos grupos mediante la prueba $\mathrm{t}$ de Student para muestras independientes, con la finalidad de comparar el estado ansiedad y depresión con el género y lugar de trabajo. Posteriormente se aplicó el coeficiente de correlación de Pearson ( $r$ ) para el análisis del estado ansiedad y depresión con el tiempo que labora. Para los análisis estadísticos antes mencionados se utilizaron mediante el programa InfoStat y SPSS 26.

\section{RESULTADOS Y DISCUSIÓN}

Al finalizar la investigación sobre el impacto psicológico estado ansiedad y depresión en los trabajadores de salud frente al covid-19 en el cantón La Troncal se obtiene resultados de gran importancia respecto a los objetivos planteados, entre ellos la edad media de la población estudiada es 34.57 años y el tiempo medio que labora dentro de la institución es 4.63 años, participó mayoritariamente el género femenino $(n=70)$, Enfermeras $(n=37)$, lugar de trabajo en iguales condiciones centro de salud tipo $C(n=50)$ Hospital Darío Machuca Palacios ( $n=50)$. En cuanto a la ansiedad está presente $(n=81)$, pero la depresión está ausente $(n=89)$.

\section{Características sociodemográficas de la población estudio}

La edad media de la población estudiada es 34.57 años y el tiempo medio que labora dentro de la institución es 4.63 años (Tabla 1). 
Tabla 1. Medidas de tendencia central de la edad y tiempo que labora.

\begin{tabular}{ccc}
\hline & EDAD & TIEMPO QUE LABORA \\
\hline Media & 34,57 & 4,63 \\
D.E. & 6,77 & 3,52 \\
Mín & 24 & 1 \\
Máx & 49 & 15 \\
Mediana & 34 & 3 \\
Q1 & 28 & 2 \\
Q3 & 40 & 7 \\
\hline
\end{tabular}

En cuanto a las características tipo $C(n=50)$ Hospital Darío Machuca Palacios sociodemográficas donde muestra que $(n=50)$. En cuanto a la ansiedad está presente el género femenino $(n=70)$ participo $(n=81)$, pero la depresión está ausente $(n=89)$ mayoritariamente, Enfermeras ( $n=37)$, lugar de (Tabla 2). trabajo en iguales condiciones centro de salud

Tabla 2. Descripción de las características sociodemográficas.

\begin{tabular}{llll}
\hline & & $\mathbf{n}$ & \% \\
\hline GENERO & Masculino & 30 & 30 \\
PROFESION & Femenino & 70 & 70 \\
& Médico & 25 & 25 \\
& Enfermera & 37 & 37 \\
& Auxiliar de enfermería & 10 & 10 \\
& Laboratorio & 12 & 12 \\
& Farmacia & 10 & 10 \\
LUGAR DE TRABAJO & Rayos X & 6 & 6 \\
& Centro de Salud tipo C & 60 \\
Ansiedad & Hospital Darío Machuca & 50 & 50 \\
& Ausencia & 50 & 19 \\
Depresión & Presencia & 19 & 81 \\
& Ausencia & 81 & 89 \\
\hline & Presencia & 89 & 11 \\
\hline
\end{tabular}


Estado ansiedad y depresión con el género y lugar de trabajo

La Tabla 3 muestra que tanto el género femenino como masculino tienen igual ansiedad y depresión, en cuanto al lugar de trabajo se evidencia que el centro de salud Tipo C tiene mayor nivel de ansiedad que el Hospital Darío Machuca Palacios, mientras que se evidencio depresión en iguales niveles en las 2 casas de salud.

Tabla 3. Análisis de las relaciones del estado ansiedad y depresión con el género y lugar de trabajo.

\begin{tabular}{lcccc}
\hline \multicolumn{5}{c}{ Género } \\
\hline & Masculino & Femenino & \\
Ansiedad & $\mathrm{m}$ & $\mathrm{m}$ & $\mathrm{t}$ & $\mathrm{p}$ \\
Depresión & 4,6 & 5,09 & $-1,38$ & 0,1705 \\
& 1,57 & 1,36 & 1,05 & 0,2948 \\
& \multicolumn{2}{c}{ Lugar de trabajo } & & \\
Ansiedad & Centro de Salud tipo C $\quad$ Hospital Darío Machuca & & \\
Depresión & 5,36 & 4,52 & 2,67 & 0,0088 \\
\hline
\end{tabular}

Tiempo que labora en la institución con la ansiedad y depresión

Se evidenció los siguientes resultados en cuanto a la correlación realizada con la prueba $r$ de Pearson, se halló entre la ansiedad y el tiempo que labora una relación negativa $(r=-0.2)$, es decir a mayor tiempo que labora dentro de la institución menor es el nivel de ansiedad (Tabla 4).

Tabla 4. Análisis del estado ansiedad y depresión con el tiempo que labora.

\begin{tabular}{cc}
\hline & TIEMPO QUE LABORA $\mathbf{r}(\mathbf{p})$ \\
\hline Depresión & $0,04(0,72)$ \\
Ansiedad & $-0,2(0,042)$ \\
\hline
\end{tabular}




\section{Discusión}

El objetivo de la investigación fue conocer el impacto psicológico estado ansiedad y depresión en los trabajadores de salud frente al covid-19 en el cantón La Troncal. A continuación, se detallará una comparación de los hallazgos obtenido con otros estudios según los tres objetivos planteados. Para el primer objetivo que constó en describir las características sociodemográficas de la población estudio la edad media de la población estudiada es 34.57 años y el tiempo medio que labora dentro de la institución es 4.63 años, el género femenino $(n=70)$ participo mayoritariamente, Enfermeras ( $n=37)$, lugar de trabajo en iguales condiciones centro de salud tipo C $(n=50)$ Hospital Darío Machuca Palacios $(\mathrm{n}=50)$. En cuanto a la ansiedad está presente $(n=81)$, pero la depresión está ausente $(n=89)$.

En un estudio realizado en China donde participaron 230 personal de salud con edades entre 20 a 59 años $(32,6 \pm 6,2)$, mayoritariamente mujeres $(n=187,81.3 \%)$, médicos $(n=70,30.4 \%)$ y enfermeras $(n=160,69.6 \%)$. La tasa de ansiedad fue del $23,04 \%$, siendo en el género femenino mayor que la de los hombres $25,67 \%$ frente $11,63 \%$. En cuanto a la profesión las enfermeras presentaron una tasa de ansiedad más alta que los médicos $26.88 \%$ y $14.29 \%$ respectivamente (7).

En Perú participaron en el estudio 91 licenciados en enfermería con edad media de 38,14 años con un mínimo de 22 años y un máximo de 64 años. Dentro de la categoría lugar de trabajo obtuvieron los siguientes resultados I-1. Puesto de Salud (19,8\%), I-2. Puesto de
Salud con médico (19,8\%), I-3. Centro de Salud sin Internamiento (15,4\%), I-4. Centro de Salud con Internamiento (12,1\%), II-1. Hospital I (4,4\%), II-2. Hospital II (6,6\%), III-1. Hospital III $(19,8 \%)$ y III-2. Instituto especializado (2,2\%), de quienes el $78 \%$ trabaja en contacto directo con pacientes infectados por COVID-19. En relación a la salud mental de los trabajadores de salud se encontró depresión (30,8\%), ansiedad $(41,8 \%)$ y estrés $(34,1 \%)(10)$.

Obtuvieron resultados similares en Italia con la participación 1379 personal de salud, en su mayoría de género femenino (77.2\%), médicos (37.64\%) y enfermeros (34.23\%), en el lugar de trabajo en primera línea (52.57\%). De esta población presentaron síntomas de depresión $(n=273,19,80 \%)$, síntomas de ansiedad $(n=114,8,27 \%)$, insomnio $(n=302$, $21,90 \%)(11)$.

Un porcentaje significativo de profesionales en Europa reportó síntomas de ansiedad $(71,6 \%)$, depresión $(60,3 \%)$ y estrés (El 14,5\%), de los participantes en el estudio fueron mayoritariamente mujeres $(73,6 \%)$, y el rango de edad se situó entre los 18 y 64 años con una media de 40.20 , y un $90 \%$ trabajaron con pacientes COVID positivo (12).

Comparando el estado ansiedad y depresión con el género y lugar de trabajo en los trabajadores de la salud en el cantón la Troncal se obtuvo que tanto el género femenino como masculino tienen igual ansiedad y depresión, en cuanto al lugar de trabajo se evidencia que el centro de salud Tipo $C$ tiene mayor nivel de ansiedad que el Hospital Darío Machuca Palacios, mientras 
que se evidencio depresión en iguales niveles en las 2 casas de salud.

Un estudio similar en profesionales sanitarios españoles mostró mayores niveles de ansiedad ( $[419]=2,66, p=0,008)$ y estrés ( $t$ $[419]=2,21, p=0,027)$ en el género femenino. El 71,5\% si había tenido contacto con personas infectadas por COVID-19 y el $44,4 \%$ indico sentir miedo al contagio, indicando niveles de ansiedad (25.3\%), depresión (19.5\%), estrés (32.1\%) e insomnio (21\%) (13).

Un metaanálisis de estudios realizados en China indicó que existen una baja calidad de sueño y altos niveles de ansiedad, depresión, somatización y síntomas del trastorno obsesivo compulsivo en los trabajadores de salud; de este grupo los menores de 35 años pero con mayor carga de trabajo hospitalario frente a la COVID-19 demostraron más síntomas de ansiedad, y sus principal temor fue infectar a sus colegas o a sus familias, la falta de medidas de protección personal y la violencia médica (14).

Un estudio en España realizado a 1422 trabajadores de la salud evidenció que el $56,6 \%$ presentaron síntomas de trastorno por estrés postraumático, el $58,6 \%$ trastorno de ansiedad, el $46 \%$ trastorno depresivo y el $41,1 \%$ se siente agotado emocionalmente. En cuanto al género, ser mujer se asocia significativa y positivamente con el estrés postraumático, ansiedad y depresión. En tanto al lugar de trabajo laborar fuera de la Comunidad de Madrid y en cualquier centro que no sea atención primaria, hospital, geriátrico o centro de día (otra categoría) se relaciona de forma negativa y significativa con el estrés postraumático. En conclusión, las variables de riesgo de ansiedad y depresión serían una persona que es mujer, que trabaja turnos de 12 o 24 horas y está preocupada de que un miembro de la familia pueda estar infectado (15).

Se evidencio en un metaanálisis que señalaron índices significativamente más altos para los trabajadores médicos de primera línea con respecto a la ansiedad, la depresión y los trastornos del sueño, todos con valores de $p<0,0001$. Por otro lado, los resultados muestran que las puntuaciones de traumatización indirecta de las enfermeras de primera línea, incluidas las puntuaciones de las respuestas fisiológicas y psicológicas, fueron significativamente más bajas que las de las enfermeras que no pertenecen a la primera línea (16).

Y al analizar el tiempo que labora en la institución con la ansiedad y depresión. Se evidenció los siguientes resultados en cuanto a la correlación realizada con la prueba $r$ de Pearson, se halló entre la ansiedad y el tiempo que labora una relación negativa $(r=-0.2)$, es decir a mayor tiempo que labora dentro de la institución menor es el nivel de ansiedad.

Una revisión sistemática sobre las mismas variables obtuvo como resultado mayor ansiedad y depresión en profesionales de salud que trabajan en primera línea, con mayor predominio enfermería (40\%), las mujeres tienen niveles más altos de ansiedad de severa a muy severa (21\%) frente a los hombres (4\%). 
En algunos estudios la edad representó un factor protector mientras que otros estudios supuso más síntomas de ansiedad y depresión. Otros factores agravantes fueron padecer enfermedades crónicas, antecedentes de problemas en la salud mental y tener menor experiencia laboral (17).

Otro estudio similar aplicado a 442 profesionales en salud de quienes tenían una experiencia laboral promedio $11.49 \pm 8.96$ años, mostró síntomas de depresión $(n=286$, $64.7 \%)$, ansiedad $(n=224,51.6 \%)$ y estrés $(n=182,41.2 \%)$, los factores asociados para dichos resultados fueron ser mujer, joven, estado civil soltera y tener menor experiencia laboral. Además, para la subescala de DAS-21 los factores asociados fueron: aumento de las horas de trabajo semanales, mayor número de pacientes atendidos con COVID-19 y menor nivel de apoyo de supervisores (18).

\section{CONCLUSIONES}

Comparando el estado ansiedad y depresión con el género y lugar de trabajo se obtuvo que tanto el género femenino como masculino tienen igual ansiedad y depresión, en cuanto al lugar de trabajo se evidencia que el centro de salud Tipo $C$ tiene mayor nivel de ansiedad que el Hospital Darío Machuca Palacios, debido que la primera casa de salud es considerada como centro asistencial COVID dentro del cantón la Troncal, mientras que se evidencio depresión en iguales niveles en las 2 casas de salud.
Y al analizar el tiempo que labora en la institución con la ansiedad y depresión se halló entre la ansiedad y el tiempo que labora una relación negativa $(r=-0.2)$, es decir a mayor tiempo que labora dentro de la institución menor es el nivel de ansiedad, la mayor parte de profesionales de salud según el transcurso de los años laborales toman cierto nivel de confianza y relajamiento en cuanto a medidas de precaución se deben tomar para la atención directa de pacientes.

La pandemia por COVID-19 ha sido sin duda uno de los mayores retos para la salud, pues el prolongamiento del mismo y el enfrentamiento hacia lo desconocido hizo que inicialmente tanto la población en general y aún más el personal que trabaja en salud sufrieran un quebrando en su salud mental, sin embargo, al pasar de los días meses e incluso años esto ha ido cambiando y nos hemos ido acostumbrado a la nueva realidad.

La base de datos obtenida dentro de este estudio es importante pues no se ha considerado dentro de otras investigaciones esta población, además de servir como pionera para futuros estudios, sería importante considerar variables como miedo al COVID-19 (19), malestar psicológico (20), estrés (21) y síntomas postraumáticos (22).

\section{REFERENCIAS BIBLIOGRÁFICAS}

1. Huarcaya-Victoria J. Consideraciones Sobre La Salud Mental En La Pandemia De Covid-19. Rev Perú Medica Exp Salud Publica. 2020;37(1):1-8. 
2. OPS. Actualizacion COVID19 Argentina abril 2021. Organ Panam Salud. 2021;3-45.

3. Molina YA, Solano YH, Hernández YC, Guyat DC, Mederos YG. Manifestaciones psicológicas frente a la situación epidemiológica causada por la COVID-19. Rev Habanera Ciencias Médicas. 2020; 19:1-13.

4. Gutierrez A, Cruz A, Zaldivaar E. Gestión de seguridad psicológica del personal sanitario en situaciones de emergencia por COVID-19 en el contexto hospitalario o de aislamiento. 2020;3704:873-6.

5. Miranda R, Murguía E. Síntomas de Ansiedad y Depresión en Personal de Salud que Trabaja con Enfermos de COVID-19. Int J Med Surg Sci. 2021;1-12.

6. Valero $\mathrm{N}$, Velez $\mathrm{M}$, Duran A, Torres M. Afrontamiento del COVID-19: estres, miedo, ansiedad y depresion. Enferm Investig Vinculacion, Docencia y Gest [Internet]. 2020;5(3):63-70. Available from: file:///C:/Users/MariaJose/OneDrive/ Escritorio/913-110-2157-1-10-20200706.pdf

7. Lozano-Vargas A. Impacto de la epidemia del Coronavirus (COVID-19) en la salud mental del personal de salud y en la población general de China. Rev Neuropsiquiatr. 2020;83(1):51-6.

8. Cahill CR. Cinco retos psicológicos de la crisis del COVID-19. Negat no Posit results [Internet]. 2020;5(6):1,2. Available from: www. cristinarodriguezcahill-psicologa.com

9. Carbonell MM, Díaz RP, Marín AR. Valor diagnóstico de la Escala de Ansiedad y Depresión de Goldberg (EAD-G) en adultos cubanos. Univ Psychol. 2016;15(1):177-92.

10. Virto-Concha Carlos Alberto Virto-Farfán Carlos Hesed Cornejo-Calderón Angela Annet3 Loayza-Bairo Wendy Álvarez-Arce Brenda Gretel Gallegos-Laguna Yván TriveñoHuamán Jahaira Jenilee. Estrés ansiedad y depresión con estilos de afrontamiento en enfermeras en contacto con Covid-19 Cusco Perú. Cusco Perú Rev RECIEN octubreNoviembre [Internet]. 2020; 9:50-60. Available from: file:///C:/Users/hp/Downloads/44-Texto del artículo-189-2-10-20201030.pdf
11. Rossi R, Socci V, Pacitti F, Di Lorenzo G, Di Marco A, Siracusano A, et al. Mental Health Outcomes among Frontline and Second-Line Health Care Workers during the Coronavirus Disease 2019 (COVID-19) Pandemic in Italy. JAMA Netw Open. 2020;3(5):2019-22.

12. Erquicia J, Valls L, Barja A, Gil S, Miquel $J$, Leal-Blanquet $J$, et al. Emotional impact of the Covid-19 pandemic on healthcare workers in one of the most important infection outbreaks in Europe. Med Clin (Barc) [Internet]. 2020;155(10):434-40. Available from: https:// doi.org/10.1016/j.medcli.2020.07.006

13. Dosil Santamaría M, Ozamiz-Etxebarria N, Redondo Rodríguez I, Jaureguizar AlbonigaMayor J, Picaza Gorrotxategi M. Psychological impact of COVID-19 on a sample of Spanish health professionals. Rev Psiquiatr Salud Ment [Internet]. 2021;14(2):106-12. Available from: https://doi.org/10.1016/j.rpsm.2020.05.004

14. Villca JL, Moreno RA, Gomez CA, Vargas AA. Influencia de la pandemia del Covid-19 en la Salud Mental de los Trabajadores en salud. Gac Medica Boliv [Internet]. 2021;44(1):75-80. Available from: http://www. gacetamedicaboliviana.com/index.php/gmb/ article/view/200

15. Luceño L, Talavera B, Yolanda G, Martín J. Síntomas de estrés postraumático, ansiedad, depresión, niveles de resiliencia y agotamiento en el personal sanitario español durante la pandemia de COVID-19. Int J Environ Res Public Health [Internet]. 2020;17(15):1-29. Available from: https://pubmed.ncbi.nlm.nih. gov/32751624/

16. da Silva FCT, Neto MLR. Psychiatric symptomatology associated with depression, anxiety, distress, and insomnia in health professionals working in patients affected by COVID-19: A systematic review with metaanalysis. Prog Neuro-Psychopharmacology Biol Psychiatry [Internet]. 2021;104(August 2020):110057. Available from: https://doi. org/10.1016/j.pnpbp.2020.110057 
17. Danet A. Impacto psicológico de la COVID-19 en profesionales sanitarios de primera línea en el ámbito occidental. Una revisión sistemática. Med Clin (Barc). 2021;156(9):449-58.

18. Elbay RY, Kurtulmuş A, Arpacıoğlu $S$, Karadere E. Depression, anxiety, stress levels of physicians and associated factors in Covid-19 pandemics. Psychiatry Res. 2020;290(May):1-5.

19. Ramírez-Coronel AA, Martínez-Suárez $P C$, Pogyo-Morocho GL, Estrella-González M de los Á, Mesa-Cano IC, Minchala-Urgilés RE, et al. Evaluación psicométrica e intervención de enfermería frente al miedo a Covid-19. Arch Venez Farmacol y Ter. 2020;39(5):660-6.

20. Gonzalez $D$, Beltrán $P$, Martínez $P$. Explorando el potencial de aprendizaje de personas con esquizofrenia en distintas condiciones psicopatológicas. Clínica y Salud. 2016; 27:81-8.
21. Monterrosa-Castro A, Dávila-Ruiz R, Mejía-Mantilla A, Contreras-Saldarriaga J, Mercado-Lara M, Florez-Monterrosa C. Estrés laboral, ansiedad y miedo al COVID-19 en médicos generales colombianos. MedUNAB. 2020;23(2):195-213.

22. Ramírez-Ortiz J, Castro-Quintero D, LermaCórdoba C, Yela-Ceballos F, Escobar-Córdoba F. Mental health consequences of the COVID-19 pandemic associated with social isolation. Colomb J Anesthesiol. 2020;48(4).

Conflicto de intereses: Ninguno declarado por los autores. Financiación: Ninguna declarada por los autores.

Agradecimiento: Ninguno manifestado por los autores

\section{ACERCA DE LOS AUTORES}

Rosa María Zambrano Garcés. Psicólogo. Magíster en Psicoanálisis con mención en Educación. Doctorante en Psicología. Docente Investigadora en Enfermería, Sicología Educativa, Educación Inicial Universidad Católica de Cuenca, Ecuador.

Enriqueta de los Ángeles Martínez Vicuña. Licenciada en enfermería. Magister en gerencia para el desarrollo local; Magister en Ginecología infanto juvenil y salud reproductiva del adolescente. Interna rotativa comunitaria, Centro de Salud Tambo; interna rotativa hospitalaria, Hospital Homero Castaier Crespo; enfermera rural, Distrito de Salud 03D01 Pindilig; Enfermera 3 operativa Hospital Darío Machuca Palacios.

Luis Cornelio Romero Mogrovejo. Doctor en Medicina y Cirugía. Abogado de los Tribunales de Justicia de la República. Magister en Medicina Forense. Director de Carrera Enfermería, Universidad Católica de Cuenca. Docente Investigador Carrera Educación Inicial y Parvularia, Derecho.

Andrea Vicuña Palacios. M. Licenciada en enfermería. Magister en gestión del cuidado. Interna rotativa comunitaria, Centro de Salud Cañar; interna rotativa hospitalaria, Hospital Homero Castaier Crespo. Enfermera rural, Distrito de Salud 06D03 Alausi-Chunchi y enfermera 3 operativa Hospital Darío Machuca Palacios. 